\title{
Integration of knowledge, visualization and dissemination of spatio-temporal data through geoportal systems
}

\author{
Stanislav Yamashkin ${ }^{1, *}$, Ekaterina Yamashkina $^{2}$, and Anatoliy Yamashkin ${ }^{1}$ \\ ${ }^{1}$ National Research Mordovia State University, 68, Bolshevistskaya Str., Saransk, 430005, Russia \\ ${ }^{2}$ MIREA - Russian Technological University, 78, Vernadskogo prospect, Moscow, 119454, Russia
}

\begin{abstract}
The article describes the basic requirements for the integration of knowledge, visualization and dissemination of spatio-temporal data through geoportal systems. Evaluation of the effectiveness of the implementation of the designed geoportal interfaces is carried out. It was shown that the main advantages of geoportals are considered the absence of the need for additional use of special programs, access at any time from any location, the ability to integrate with third-party websites, etc. The proposed structure of the platform solution is determined by the hypothesis that in order to optimize the storage processes and practical use of spatial data, a project-oriented spatial data infrastructures (SDIs) should contain loosely coupled and strongly connected storage subsystems, analysis and synthesis, as well as visualization and dissemination of spatial data; external objects in relation to SDI should be key actors, as well as external consumers and thirdparty providers of spatial data and information. Geoportal systems are the external part of the SDI and are used to disseminate and visualize the accumulated geospatial information and analysis results during design work. Solving the main issues of creating the infrastructure of geoportals makes it possible to create a successful strategy for the sustainable development of regions.
\end{abstract}

\section{Introduction}

The main advantages of geoportals are considered the absence of the need for additional use of special programs, access at any time from any location, the ability to integrate with third-party websites, etc.

The interfaces of geoportals, with the help of which there is an interaction with users, usually have high quality and functional requirements [1].

Existing developments touch upon the issues of using geoportals, improving User Experience (UX): the perception of user actions with the interface. This article will consider in detail the problems of visualization and distribution of geodata: design and research of stable, adaptive, convenient geoportal interfaces.

Such interfaces can help solve the problem of geospatial dissemination. Also in this work, a solution to the problem of visualizing geodata is proposed. The quality of geoportals

\footnotetext{
* Corresponding author: yamashkinsa@mail.ru
} 
depends on user interfaces. The study of the available geoportal solutions made it possible to develop a list of basic requirements: flexible design process and interface research; crossplatform, using your own software modules. This list of requirements can be supplemented or changed depending on the tasks.

Solving the main issues of creating the infrastructure of geoportals makes it possible to create a successful strategy for the sustainable development of regions.

The article describes the basic requirements for the integration of knowledge, visualization and dissemination of spatio-temporal data through geoportal systems. The effectiveness of the implementation of the designed geoportal interfaces is also assessed. Solving the main issues of creating the infrastructure of geoportals makes it possible to create a successful strategy for the sustainable development of regions.

\section{Materials and Methods}

\subsection{Requirements for visualization and geospatial data interfaces}

The main advantages of geoportals are considered the absence of the need for additional use of special programs, access at any time from any location, the ability to integrate with thirdparty websites, etc.

The interfaces of geoportals, with the help of which there is an interaction with users, usually have high quality and functional requirements [1].

Recognizing the rationality of the procedure for studying the interfaces of geoportal solutions and the need to form a list of requirements for them, we will identify the basic requirements for visualization and dissemination of geospatial data:

1) Flexible interface design and research process. The essence of this requirement lies in the process of analyzing the advantages and disadvantages of geoportals, their capabilities and competitiveness. In the rapidly growing web environment, the importance of the risk management skills required for sustainable development cannot be denied. The waterfall model of the life cycle, proposed by Winston Royce [13], assuming that the process of forming software solutions is a flow, in this case, in the field of researching geoportals, is no longer relevant. The desire to initially think through all functional and quality requirements without exception is doomed to failure in the context of a continuously changing information society, and finding a solution often rests on a flexible process aimed at iterative planning, risk management, dynamic development of conditions. Thus, even several years before this work was written, there was little research devoted to the creation of adaptive cross-platform geoportal interfaces. Nowadays, this concept is considered the benchmark and has a focus on flexible development processes that allow you to quickly and easily move the plan to the latest interface solutions.

2) Focus on the future. In keeping with the requirements, geoportal interfaces must be improved in the future. So, technological processes of virtual reality developing in our time, in the near future, can change the image of current GIS, as it happened earlier with the introduction of mobile adaptive interfaces. At the same time, with this, the Internet of Things promises tremendous opportunities, being actively implemented in all areas of life. In this context, geoportal solutions will be the main link for interaction with smart devices based on the concept of Machine-to-Machine, M2M. Their cartographic interfaces should implement monitoring and supervision of the functioning of smart devices.

3) Human-centered interface design is an important policy, assuming that at any time during research, attention is paid to user needs. Geoportal design should be analyzed in the course of researching the user's actions (the period spent in the system, the map of transitions, scrolling, moving the mouse cursor). 
4) The need for cross-platform adaptive interfaces. The widespread use of smartphones and tablets forms its own requirements for graphical interfaces of geoportals. Pages and interface elements of web systems must be adapted to the display resolution of mobile devices.

5) The advantage of using your own software modules over existing frameworks. In order to achieve maximum efficiency and avoid vulnerabilities in the created geoportal system, it is important to use your own software modules, in particular, this requirement is especially important for the functioning of the server part.

The geoportal of the Russian Geographical Society in the Republic of Mordovia functions in a similar way - it is a social and information project aimed at raising public awareness of the problems of preserving the natural, historical and cultural heritage of the Russian region.

Figure 1 shows the structural diagram of the geoportal, taking into account the designed pages and interfaces.

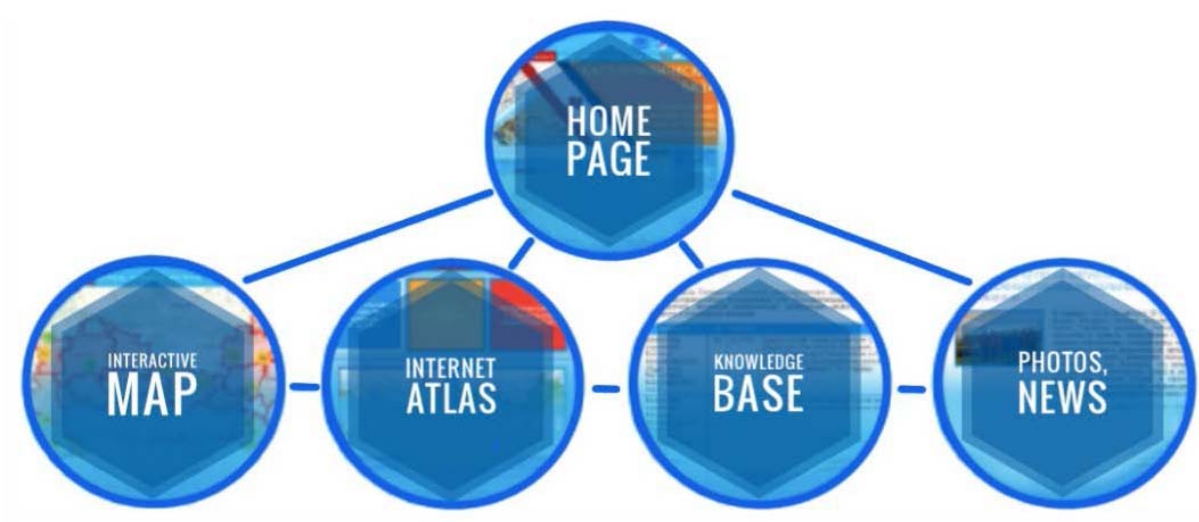

Fig. 1. Structural diagram of a geoportal solution.

\subsection{Requirements for the dissemination, structure and protection of geospatial data}

The solution of urgent problems in the field of land use, strategic planning, modeling and forecasting of spatio-temporal processes should be based on a project-oriented spatial data infrastructure. Analysis of the history of development and the current state of the issue of designing platform solutions in the designated area made it possible to determine the basic architectural components of the SDI, indicated on the structural component diagram.

While the design, development, implementation and use of a project-oriented SDI should be based on specific goals and objectives, the proposed architectural solution is a basic system that can be decomposed and expanded for a specific problem area.

The structure of the platform solution is determined by the hypothesis that in order to optimize the storage processes and practical use of spatial data, a project-oriented SDI should contain loosely coupled and strongly connected storage subsystems (cloud or local storage), analysis and synthesis, as well as visualization and dissemination of spatial data (in the form of geoportal systems); external objects in relation to SDI should be key actors (users of geoportal systems, scientists and specialists), as well as external consumers and third-party 
providers of spatial data and information.

Geoportal systems (geoportals) are the external part of the SDI and are used to disseminate and visualize the accumulated geospatial information and analysis results during design work. Geoportals are a special case of a web portal and their central task is to represent spatial data within the framework of web interfaces accessible via the Internet.

Spatial data infrastructures play an important role in linking and integrating distributed data and systems. They facilitate the discovery, access, management, distribution and reuse of digital geospatial resources. Spatial data infrastructures also make it possible to unify access to distributed resources using web services and interfaces. However, this requires the interaction of the connected components, and the challenge is to access them using open, standardized and secure interfaces.

An infrastructure focused on the development of smart cities is proposed for development. A key aspect of the framework is that it takes into account the various components of smart city scenarios, such as city population, sensors and devices, applications, modeling and analysis tools, geographic information including information about buildings, landscape, etc.

The data infrastructure provides a well-defined structure for classifying different components and isolating common data. This distinguishes this framework from SDI, therefore, it is a service-oriented architecture and allows you to model the interaction of distributed data and systems using well-defined interface models based on geospatial consortium standards.

The structure of the geoportal implements the concept of ensuring the security of distributed applications and services in spatial data infrastructures for smart cities. The concept ensures confidentiality, security and controlled access for all interested parties and related components through the creation of reliable authorization and authentication mechanisms.

This approach simplifies the use of authentication with the latest security concepts such as OAuth2 access tokens, OpenID Connect user authentication, and Security Claims Markup Language (SAML). The infrastructure is developed on the basis of open international national standards OGC, containing data for semantic 3D models of cities and for identifying sensors and IoT devices. OGC also provides a supported framework for various web services, such as a Web Functions Service (WFS) for retrieving CityGML objects and other objectoriented datasets, a Sensor Observation Service (SOS), and a Sensor-API for retrieving realtime data from sensors. time, a directory service that allows the registration and discovery of registered resources using standardized metadata. The use of international standards allows the various components to be linked in a stable manner.

Based on the structure of a smart city, a specific set of requirements for the development of geoportal infrastructures is determined. The basic requirements are listed below.

1) Different stakeholders. Typically, smart city infrastructures include distributed systems that are used by different stakeholders (end users) such as citizens, municipalities, utility and transport providers, real estate firms, etc. It is important that the infrastructure takes into account the needs and requirements of different stakeholders and, as a result, not all data can and will be stored in one system.

2) Distributed applications. Applications should be able to register and interact with stakeholders. These applications typically use different datasets, readings, or simulations.

3) Modeling and analytical tools. They are software components designed for specific scenarios, such as estimating energy demand or production potential for buildings that simulate traffic and pedestrian flows. The results of these simulations can not only be provided to applications, but can also be used for planning and forecasting. Also, the simulation results can be used by multiple applications or one application. Consequently, such modeling tools must be registered and run separately.

4) Sensors and the Internet of Things (IoT). These devices are integral parts of several 
smart city infrastructures. They can be stationary, such as meters for weather stations. Some sensors can be mobile, such as air quality sensors. It is important to enroll such sensors and IoT devices in an infrastructure that allows their readings to be integrated with applications using analytic tools.

5) Inclusion of geographic information. All smart city concepts focus on information and communication technologies (ICT) such as the Internet of Things, big data, cloud computing, etc. However, it is important to consider geographic information as a key element [2]. City planning scenarios need to work correctly with physical reality models - 3D city models. These 3D city models represent both spatial and semantic information - the connection of physical objects such as buildings, roads, bodies of water, etc. In addition, semantic 3D city models allow interactive and spatially semantic queries and aggregates. It is important to consider other geographic data such as maps and terrain as well as building information models (BIM).

6) Interoperability. In order to process heterogeneous data, applications, sensors and IoT devices must interact with various connected components and systems. Interoperability facilitates access and retrieval of data, services and applications using standardized and stable interfaces [3].

7) Related components. The use of standardized interfaces such as OGC also allows you to manage and access various components that are related to each other. Working with such related components is also an important requirement for a distributed system. For example, if a smart meter is installed in a building, a web service (such as a sensor monitoring service) can retrieve semantic information about the building (in this case, to measure gas flow in real time).

8) Authentication and authorization. The most basic requirement is to secure access to data and functions. Thus, user authentication and authorization plays an important role. Username and password are usually used for authentication. Authorization describes the process of checking whether a user has access rights to a specific resource. Modern standards such as OAuth2 allow you to enable delegation of access from the resource owner (that is, the user) to a trusted application and access protected resources without exposing credentials. The technology uses access tokens. OAuth2 is considered the most advanced technology for web and mobile applications and is supported by many applications [4].

9) User information. Another important aspect is user privacy. The OpenID Connect standard was designed as an extension of the OAuth2 platform to provide the ability to associate a user with access tokens. The implementation using OpenID Connect provides easy integration with external authentication services such as Google and Facebook, which make the application convenient for authenticating multiple users around the world.

10) Single sign-on with delegated authorization. Modern standards such as OAuth2 already support closed authorization, which allows a trusted application to access a protected resource without exposing basic credentials [5].

Based on the requirements described above, listed in the previous section, you must use OAuth2 access tokens for security. Because users click on the link to get information, an access token must be obtained either as part of the URL (the access_token parameter) or as part of an HTTP header named authorization. Once the access token is isolated from the incoming request, it needs to be verified and authenticated by the authorization server (AS) that issued the token. If the AS has successfully verified the access token, it can exercise access control based on the token metadata. This means that any authenticated user can get the information they need. 


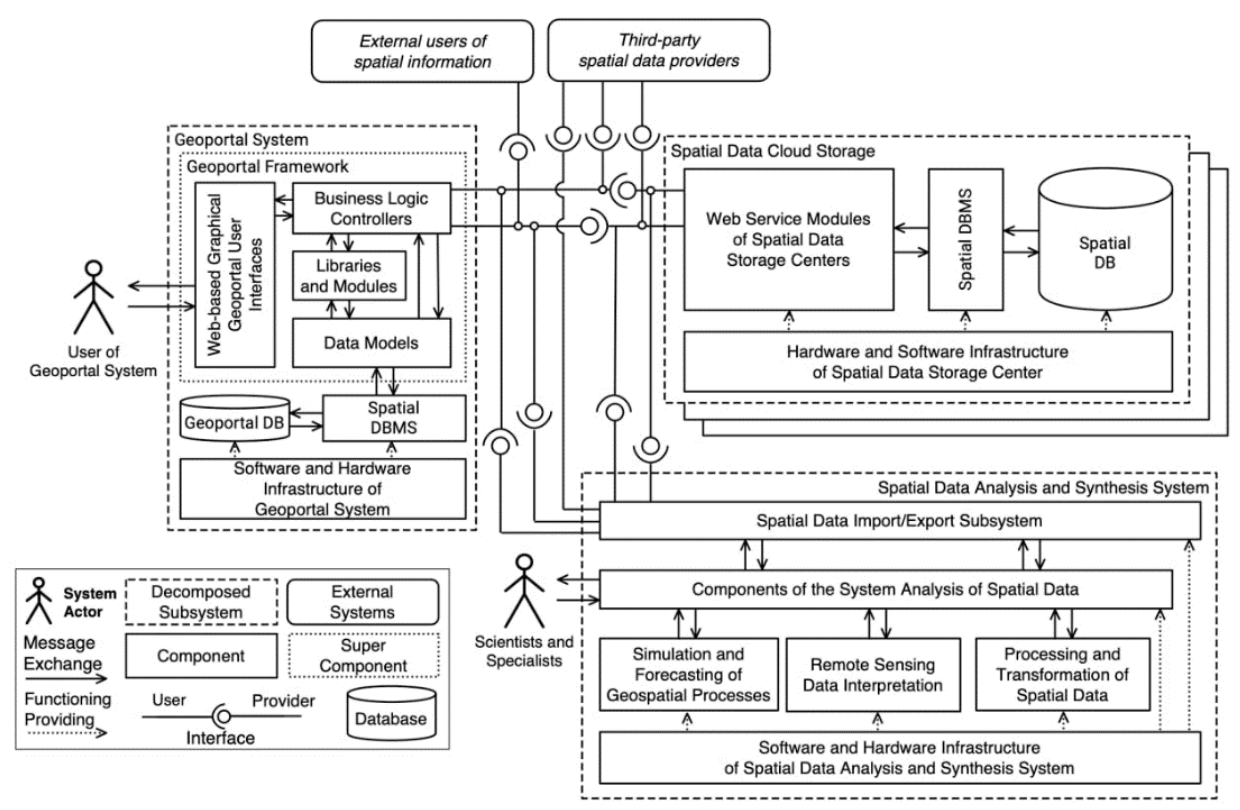

Fig. 2. Infrastructure diagram of a geoportal solution.

Thus, based on the proposed data infrastructure, a new concept of data access protection and integration of distributed applications is presented. The approach responds to identified key requirements and uses integration with modern security standards such as OAuth, SAML and OpenID Connect. Development data is used at the stage of system deployment.

As AI-based geoportal solutions become more widespread and valuable, they are increasingly targeted by hackers. Attempts are made to unauthorized access and theft of models (for example, through the introduction of malware or code injection). Such copyright infringement can jeopardize intellectual property.

Therefore, it is also very important to obtain ownership of the software models in order to protect them. It is also necessary to timely detect attacks on geoportal systems and repel them.

\section{Results}

The proposed new technologies for data analysis in the formation of spatial information infrastructure are based on the use of a system of methods of mathematical and geoinformation modeling, ensemble learning. The purpose of their implementation is to increase the efficiency of geodiagnostics and mapping of spatio-temporal changes in the geosystems of regions.

In the previous sections of this work, a system of recommendations for visualization and the interface part of the architecture of the geoportal system is described, which will integrate the spatio-temporal structure of the natural-social-production systems (NSPS) and improve the accuracy and efficiency of identifying the patterns of structure, dynamics and development of geosystems, predicting the direction and intensity of ecological-socioeconomic development regions.

The task of forming information resources for the purposes of optimizing the process of economic development largely depends on the object of research, its parameters, structure, and the nature of connections between elements. The concept of a cultural landscape, taken as a basic one, is implemented with the complementarity of geoecological and cultural 
approaches, positioning the studied area as a result of the interaction of natural, social, production systems, historical, cultural, environmental and other factors.

Research of analytical aspects of the territorial organization of the processes of economic development of landscapes based on information methods. At this stage, the following results will be obtained:

1) identified natural, social and economic factors in the formation of factorial-dynamic series of cultural landscapes;

2) analyzed the territorial, functional and hierarchical structures of territorial SSPS, studied their individual components and the relationship between them;

3) a component-wise and integral assessment of the economic development of landscapes at different territorial levels was carried out.

At the stage of synthesizing the results of analytical studies, the following methodological principles and methodological techniques will be developed:

1) spatio-temporal systematization of significant data sets of multispectral satellite images using GIS technologies;

2) the spatio-temporal patterns of formation, functioning and development of territorial NSPS were revealed;

3) approaches have been developed for mapping the NSPS at the regional and local levels of the organization for the purpose of optimizing the economic development of landscapes;

4) the structure of the regional geoportal has been worked out for making management decisions in the field of optimizing the economic development of the territory.

Interdisciplinary studies of the NSPS of modern landscapes include geographic, landscape and ecological approaches. In practical terms, the application of these approaches makes it possible to map resources at the regional and local levels of economic development of the territory.

When performing this work, the mathematical apparatus of the system being created was developed and optimized (including the method of analyzing the geophysical envelope using neighborhood descriptors and an algorithm for analyzing the structure of lands using ensemble systems that allow combining several classifiers and making an informed decision on the choice of the resulting hypothesis), which is the basis for creating an automated system for analyzing remote sensing data for monitoring the state of land and predicting natural spontaneous processes. In comparison with the previous stage, it was possible to increase the accuracy of the methods by $5 \%$ due to the elimination of defects in the program code of the system.

The developed algorithms and techniques are implemented in the form of an optimized software package. The created software package has been tested and optimized when solving the problem of decoding a system of test sites for analyzing space images and recognizing spatial objects on the territory of the Republic of Mordovia. The approbation of the program for the analysis of the geophysical envelope at various test sites showed a variation in the classification accuracy within 91-98\%, taking into account the neighborhood descriptors.

In order to create an effective means of visualization and dissemination of spatial data, an integrated approach to the formation of the architectures of geoportal systems with intuitive user interfaces was developed.

Taking into account the descriptors of the neighborhood of different radii made it possible to increase the final classification accuracy by $8 \%$, in particular, made it possible to identify residential landscapes by analyzing the uniqueness of the texture of the neighborhood.

This area of concern should separately consider Application Programming Interface (API) and Graphical User Interface. The first class of interfaces enables automatic or automated interaction with GIS, including remote control of the system and data exchange. To maximize the efficiency of the designated processes, specialized formats and standards for representing geodata (GeoJSON, TopoJSON, KML) are used, deployed based on such 
architectural styles of interaction of components as REST, subsystems for the functioning of Web API and web services.

The described principle of building geoportal systems is implemented in one of our projects related to the processing of geospatial data, Lifeshot.info. It focuses on providing convenient cartographic interfaces to news information, and not to spatial objects, as is traditionally done. In terms of the organization of the control blocks, more attention is focused on the cartographic basis (Figure 3.4) than in the previous example. The control components are formed in such a way as to provide the ability to obtain information about current news and events taking place around the user.

Lifeshot.info approach to displaying news information is unique and fundamentally different from the solutions used by leading news vendors. The novelty and effectiveness of the result was obtained due to the fusion of the potential of geoportal systems and news resources. When developing a news geoportal, the property of adaptability of graphical interfaces was also implemented: it is convenient to receive information about surrounding events both from a desktop computer and a mobile device. Currently, a prototype of the Lifeshot.info native geoportal application for the Android operating system is being developed.

The project describes new technologies for the analysis and dissemination of spatial data in the formation of spatial information infrastructure. They are based on the use of a system of methods of mathematical and geoinformation modeling, ensemble learning. The practical purpose of their implementation is to increase the efficiency of geodiagnostics and mapping of spatio-temporal changes in the geosystems of regions.

Thus, in this section, the effectiveness of the implementation of adaptive geoportal interfaces is assessed, which allows us to make the statement that the measures taken will lead to a positive growth in traffic on the project.

\section{Conclusion}

Well-designed interfaces to geo-portal systems can help solve the problem of geospatial dissemination. Also in this work, a solution to the problem of visualizing geodata is proposed. The quality of geoportals depends on user interfaces.

The study of the available geoportal solutions made it possible to develop a list of basic requirements: flexible design process and interface research; cross-platform, using your own software modules. This list of requirements can be supplemented or changed depending on the tasks.

Solving the main issues of creating the infrastructure of geoportals makes it possible to create a successful strategy for the sustainable development of regions.

Recognizing the rationality of the procedure for studying the interfaces of geoportal solutions and the need to form a list of requirements for them, we will identify the basic requirements for visualization and dissemination of geospatial data:

1) flexible process of designing and researching interfaces;

2) focus on the future;

3) Human-centered design of interfaces;

4) The need for cross-platform adaptive interfaces;

5) The advantage of using your own software modules over existing frameworks.

The geoportal of the Russian Geographical Society in the Republic of Mordovia functions in a similar way - it is a social and information project aimed at raising public awareness of the problems of preserving the natural, historical and cultural heritage of the Russian region.

Based on the proposed data infrastructure, the concept of data access protection and integration of distributed applications is presented. The approach responds to identified key requirements and uses integration with modern security standards such as OAuth, SAML and 
OpenID Connect.

As geo-portal solutions powered by artificial intelligence become more widespread and valuable, it is also very important to obtain ownership of software models in order to protect them. It is also necessary to timely detect attacks on geoportal systems and repel them.

The project describes new technologies for the analysis and dissemination of spatial data in the formation of spatial information infrastructure. They are based on the use of a system of methods of mathematical and geoinformation modeling, ensemble learning. The practical purpose of their implementation is to increase the efficiency of geodiagnostics and mapping of spatio-temporal changes in the geosystems of regions.

An assessment of the effectiveness of the implementation of adaptive geoportal interfaces was also carried out, which allows making the statement that the measures taken will lead to a positive growth in traffic on the project.

\section{Acknowledgement}

The reported study was funded by RFBR, project number 20-37-70055.

\section{References}

1. Q. Thao Dang, P. Jasovska, H. Rammal, Journal of World Business 55(1) (2020) DOI: 10.1016/j.jwb.2019.101042

2. J. Doran, N. Doran, M. O’Connor, Cogent Economics \& Finance 6, 1442093 (2018) DOI: $10.1080 / 23322039.2018 .1442093$

3. K. Konrad, K. Böhleb, Futures 109, 101-107 (2019) DOI: 10.1016/j.futures.2019.03.003

4. F. Cosenz, G. Noto, Long Range Planning 51(1), 127-140 (2018) DOI: 10.1016/j.lrp.2017.07.001

5. D. Selloni, CoDesign for Public-Interest Services, 177-189 (2017) DOI: 10.1007/978-3319-53243-1_11

6. M. Edwards-Schachter, International Journal of Innovation Studies 2(2), 65-79 (2018) DOI: $10.1016 /$ j.ijis.2018.08.004

7. A. Ustundag, E. Cevikcan, Industry 4.0: Managing. The Digital Transformation (2017)

8. O. Kadyrova, T. Ksenofontova, R. Smirnov, IJRTE 8(2), $4302-4305$ (2019) DOI: 10.35940/ijrte.B2821.078219

9. Z. Acs, S. Estrin, T. Mickiewicz, et al., Small Business Economics 51(2), 501-514 (2018)

10. N. Golubetskaya, T. Kosheleva, V. Kunin, IOP Conference Series: Earth and Environmental Science 90(1) (2017) DOI: 10.1088/1755-1315/90/1/012049

11. A. Armisena, A. Majchrzak, Business Horizons 58(4), 389-399 (2015) DOI: 10.1016/j.bushor.2015.03.004

12. G. Morunova, S. Gorbushina, V. Okrepilov, S. Kuzmina, MATEC Web of Conferences 239, 08005 (2018) DOI: 10.1088/1757-899X/497/1/012134 